\title{
Multistaged, multidirectional strategy for safe removal of large meningiomas in the pineal region
}

\author{
Naoki Otani, MD, PhD, Kentaro Mori, MD, PhD, Kojiro Wada, MD, PhD, Arata Tomiyama, MD, PhD, \\ Terushige Toyooka, MD, PhD, and Satoru Takeuchi, MD, PhD
}

Department of Neurosurgery, National Defense Medical College, Tokorozawa, Saitama, Japan

\begin{abstract}
OBJECTIVE Pineal region meningiomas are rare and tend to be discovered only after they grow. Several simultaneous multidirectional approaches performed as a single operation have been proposed, but the best strategy to remove these deeply situated large meningiomas involving the deep vital venous system remains to be established. The authors advocate a multistaged, multidirectional approach to safely remove these challenging tumors.
\end{abstract}

METHODS Four consecutive cases of meningioma in the pineal region were treated between April 2013 and June 2016. The 3 large (> $40 \mathrm{~mm}$ diameter) tumors were removed via multistaged, multidirectional approaches (2 surgeries in $2 \mathrm{pa}-$ tients and 3 surgeries in 1 patient) with gravity retraction of the occipital or parietal lobe. The large occipital skin incision extending bilaterally was used for the next operation from the contralateral side. Combinations of the occipital transtentorial approach with or without the transfalcine approach, occipital bitranstentorial/falcine approach, combined supra-/ infratentorial transsinus approach, and contralateral parietal interhemispheric transcallosal approach were used.

RESULTS Transient visual field deficits occurred after 2 of the 8 operations, but all tumors were removed grossly or subtotally without permanent surgery-related morbidity. The galenic venous system and straight sinus remained intact in all patients. During the follow-up period (mean 29.5 months [range 13-52 months]), there were no recurrences after the final operation.

CONCLUSIONS A multistaged, multidirectional strategy with an intentional large occipital scalp incision and gravity retraction of the occipital lobe is a good choice for the safe removal of large meningiomas in the pineal region.

https://thejns.org/doi/abs/10.3171/2017.12.FOCUS17602

KEY WORDS meningioma; pineal region; multistaged operation

$\mathrm{M}$ ENINGIOMAS of the pineal region are relatively rare and account for $6 \%-8 \%$ of the pathologies in this location. ${ }^{13,19}$ The tumor originates around the midline posterior tentorial incisura at the falcotentorial junction, the velum interpositum of the posterior third ventricle, and the arachnoid membrane over the vein of Galen and generally extends downward to occupy the pineal region to the quadrigeminal cistern, with the mass effect compressing the surrounding neurovascular structures, such as the pineal body, splenium, midbrain tectum, thalamus, vein of Galen, internal cerebral vein, basal vein of Rosenthal, and straight sinus. ${ }^{2,4,6,11}$ Meningiomas of the pineal region have a benign, slow-growing nature and do not cause ocular movement disorders like malignant tu- mors of the pineal region; however, the tumor is usually quite large at the time of discovery, with or without obstructive hydrocephalus. ${ }^{15}$

Multiple compartments formed by the firm dural structures and involvement of the vital deep venous system present difficulties in the removal of these tumors, especially when they are large. The supracerebellar infratentorial approach, ${ }^{2,11}$ combined supratentorial and infratentorial approach, ${ }^{16}$ combined supra-/infratentorial-transsinus approach, ${ }^{12,18}$ unilateral occipital transtentorial approach (OTA) with or without the transfalcine approach, ${ }^{2,4,12}$, ${ }^{14,15}$ and occipital bitranstentorial/falcine approach ${ }^{5,8}$ have been advocated to remove this challenging meningeal tumor. Simultaneous multidirectional approaches, such as 
the combined supra-/infratentorial-transsinus approach and occipital bitranstentorial/falcine approach, may be advantageous in the removal of large pineal region meningiomas during a single operation, but blind spots remain around the skeletonized straight sinus and galenic venous system. ${ }^{15}$

Simultaneous bilateral retraction of the occipital lobes carries the risk of cortical blindness, and deep venous injury may result in formidable morbidity. ${ }^{4,9,16}$ Given that the meningioma is biologically benign, surgical damage to the surrounding vital neurovascular structures around a pineal region meningioma should be avoided by not adopting an aggressive simultaneous, multidirectional strategy but rather a multistaged, multidirectional strategy. However, the use of a multistaged strategy for large meningiomas in the pineal region has not been reported. In particular, the best approach and strategy to remove deeply situated meningiomas involving the vital deep venous system have not been evaluated.

Recently, we treated 4 consecutive pineal region meningiomas with sizes ranging from small $(31 \mathrm{~mm})$ to large $(57 \mathrm{~mm})$ and suggest that the multistaged, multidirectional strategy is an excellent choice for the safe removal of this challenging tumor, especially large ones ( $>40 \mathrm{~mm}$ ).

\section{Methods}

Between April 2013 and June 2016, pineal region meningiomas in 4 patients were treated via surgical removal by the senior surgeon (K.M.). MRI with gadolinium and 3D CT angiography and venography were performed for pre- and postoperative examinations. The lateral semiprone position was used for gravity retraction of the occipital lobe. An occipital horseshoe scalp incision extending bilaterally was intentionally made to prepare for the next operation from the contralateral side. A right-sided OTA was chosen to remove the tumor in the first operation in 3 of the 4 patients. During the first operation, tumor removal was stopped as soon as any difficulty was encountered in observing the tumor in the contralateral side or detaching the tumor from the neurovascular structures. One small tumor was removed through a single operation, and 3 large tumors ( $>40 \mathrm{~mm}$ in maximum diameter) were removed a few months later during either a second or third operation, which was performed from the contralateral side; different approaches were used to grosstotally remove the tumor. In this series, combinations of the OTA with or without the transfalcine approach, occipital bitranstentorial/falcine approach, combined supra-/infratentorial-transsinus approach, or contralateral parietal interhemispheric transcallosal approach were used for the multistaged, multidirectional strategy. These methods are described elsewhere. . $^{3,8,10,14,20,21}$

\section{Summary of Cases \\ Single-Staged Operation Case 1}

A 75-year-old man had a 3-month history of gait disturbance. The patient underwent surgery at a local hospital via a supracerebellar infratentorial approach, but only a biopsy specimen was obtained. After the operation, the patient's gait disturbance worsened and cognitive impairment occurred due to obstructive hydrocephalus. A ventriculoperitoneal shunt was placed and the patient's cognitive function improved, but his gait disturbance gradually worsened, and he began to have headaches, vertigo, and nausea. At this point, the patient presented to our institution. On admission, he exhibited right-sided truncal ataxia and dysdiadochokinesia and experienced headache and nausea. MRI showed a tumor in the pineal region $(31 \mathrm{~mm}$ in maximum diameter), which was located mostly in the infratentorial space and compressed the quadrigeminal plate and the anterior cerebellar vermis inferiorly. The vein of Galen was slightly displaced anteriorly. Peritumoral cyst was seen in the vermis (Fig. 1A and B). A right OTA was performed with the patient in the lateral semiprone position. The tumor was attached to the midline tentorial incisura and extended to the inferior surface of the straight sinus. The tumor was totally removed without difficulty (Fig. 1C and D). The histological diagnosis was atypical meningioma (Ki-67 5\%, WHO grade II). The patient's headache and nausea disappeared, and his gait disturbance improved (modified Rankin Scale [mRS] score $0)$. MRI showed no tumor recurrence at 1 year after the operation.

\section{Double-Staged Operation}

\section{Case 2}

A 47-year-old woman presented with headache caused by a small tumor $(13 \mathrm{~mm})$ in the posterior lower falx that was detected by MRI. Ten years later, follow-up MRI showed that the tumor in the pineal region had enlarged (41 $\mathrm{mm}$ in maximum diameter). The patient had no symptoms. The bilateral internal cerebral veins and vein of Galen were remarkably displaced downward (Fig. 2A and B). The first operation was conducted using the right occipital bitranstentorial/falcine approach with the patient in the lateral semiprone position. The tumor was attached to the falcotentorial junction; it was extremely hard and bled and was diagnosed as a fibrous meningioma (Ki-67
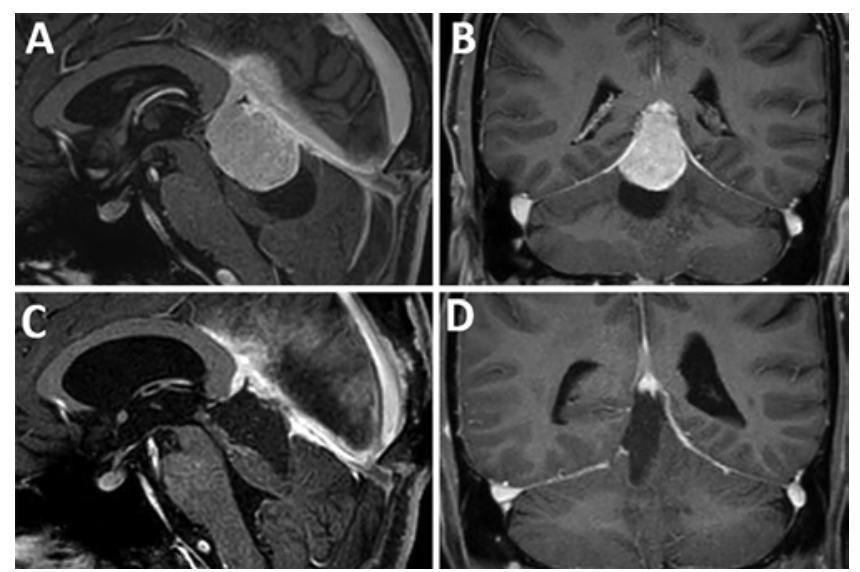

FIG. 1. Case 1. Sagittal (A and C) and coronal (B and D) Gd-enhanced T1-weighted MR images. A and B: Preoperative images showing a pineal region meningioma (maximum diameter $31 \mathrm{~mm}$ ). C and D: Postoperative images showing gross-total removal of the tumor via the right OTA. 

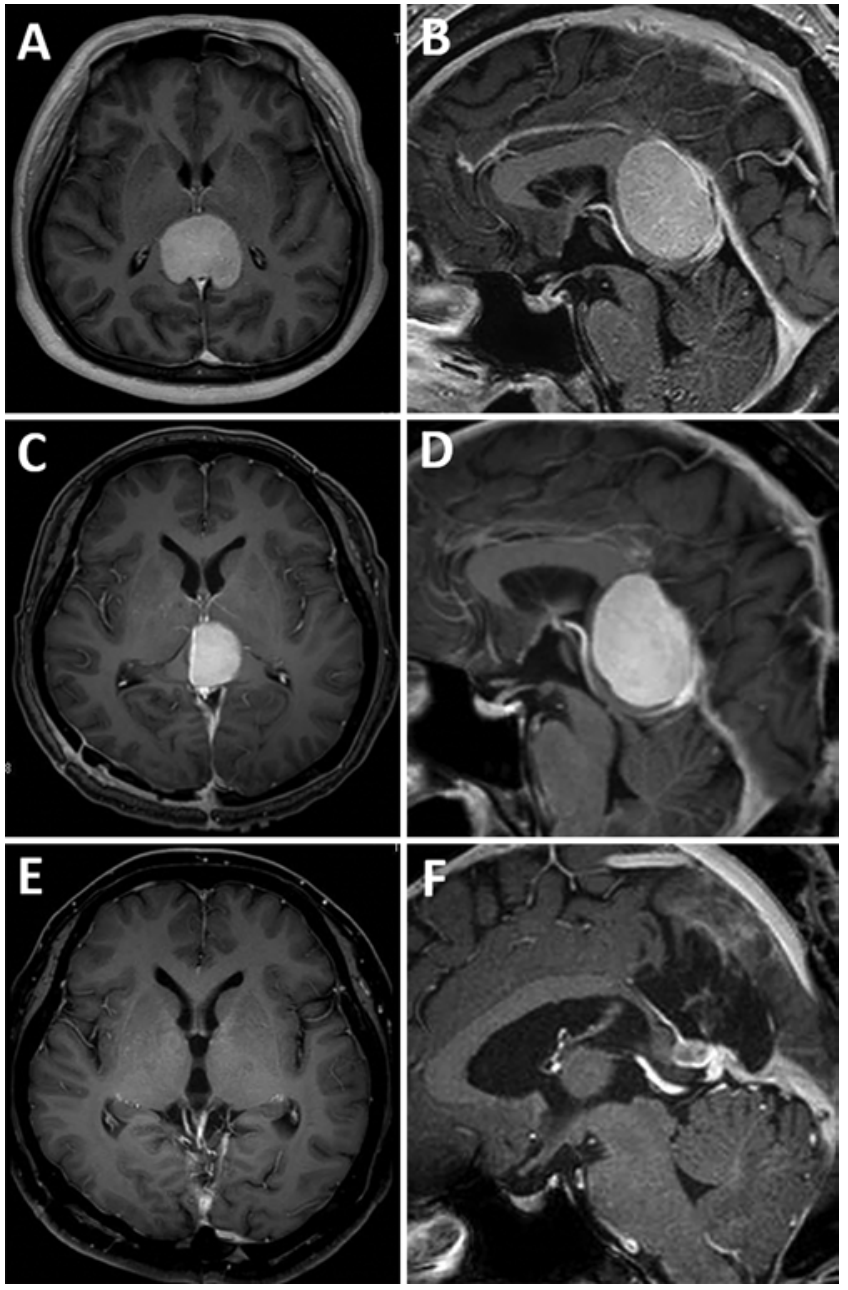

FIG. 2. Case 2. Axial (A, $C$, and $E)$ and sagittal $(B, D$, and $F) G d$ enhanced T1-weighted MR images. A and B: Preoperative images showing a pineal region meningioma (maximum diameter $41 \mathrm{~mm}$ ). C and D: Postoperative images showing that half of the tumor has been removed through the right occipital bitranstentorial/falcine approach. E and F: Postoperative images showing gross-total removal of the tumor via the left OTA

4\%, WHO grade I). Postoperative MRI showed that the right side of the tumor had been removed (Fig. $2 \mathrm{C}$ and D). The patient exhibited transient left lower homonymous quadrantanopia. After confirming disappearance of the quadrantanopia, the second operation was performed 4 months later via the left OTA with the patient in the lateral semiprone position. The tumor appeared whitish and bled less than during the first operation, because the tumor had been well detached from the falx and tentorium during the first operation. Gross-total removal was accomplished without difficulty (Fig. 2E and F). The postoperative course was uneventful (mRS score 0 ). MRI showed no recurrence 4 years later.

Case 3

A 65-year-old man had begun to experience gait disturbance, cognitive impairment, and urinary incontinence 6 months prior to referral to our institution. MRI showed a
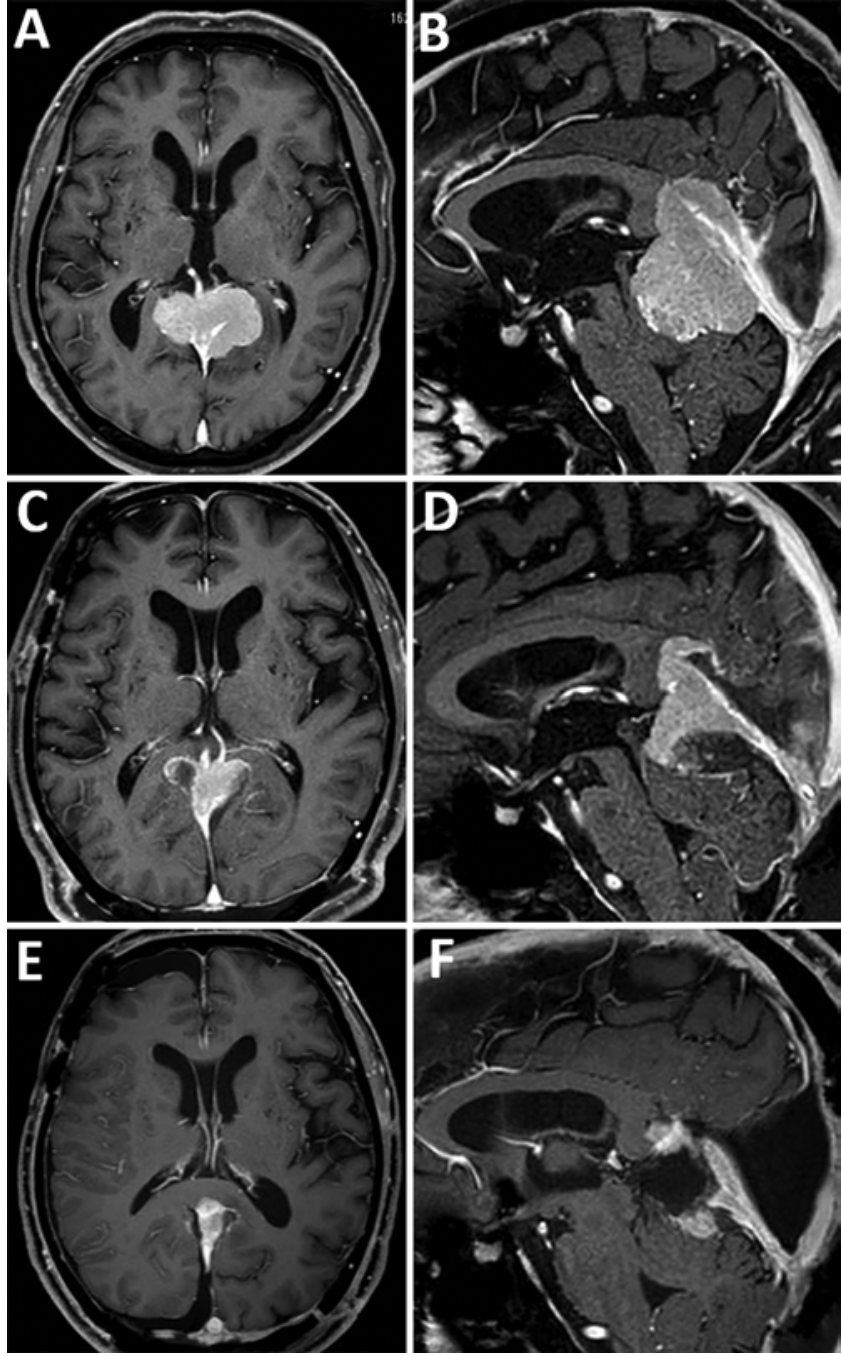

FIG. 3. Case 3. Axial (A, $C$, and $E$ ) and sagittal (B, D, and F) Gd-enhanced T1-weighted MR images. A and B: Preoperative images showing a pineal region meningioma (maximum diameter $54 \mathrm{~mm}$ ). $\mathrm{C}$ and D: Postoperative images showing a tumor remnant around the galenic system and straight sinus after removal through the combined supra-/ infratentorial left transsinus approach. E and F: Postoperative images showing subtotal removal of the tumor through the right occipital bitranstentorial/falcine approach.

large pineal region tumor (54 $\mathrm{mm}$ in maximum diameter) and hydrocephalus due to midbrain compression. The tumor mainly extended into the infratentorial space and partially into the supratentorial space, engulfing the inferior sagittal sinus, vein of Galen, and straight sinus (Fig. 3A and B). The vein of Galen was slightly displaced upward. The bilateral internal cerebral veins showed engorgement but no collateral venous flow. After an endoscopic third ventriculostomy, the patient's symptoms improved. The first operation was performed via the combined supra-/ infratentorial left transsinus approach with the patient in the lateral semiprone position (left side down) because the left transverse sinus was not dominant. The tumor was detached from the left side of the falx. However, the tumor could not be observed well from the right occipital inter- 

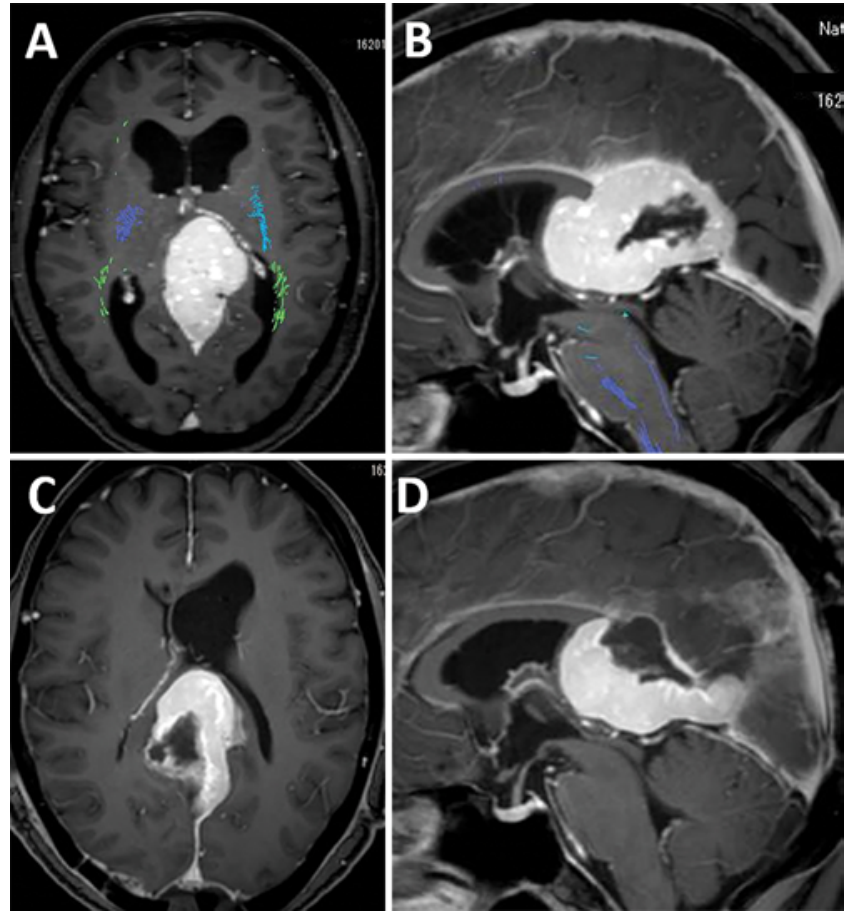

FIG. 4. Case 4. Axial (A and C) and sagittal (B and D) Gd-enhanced T1-weighted MR images. A and B: Preoperative images showing a pineal region meningioma (maximum diameter $57 \mathrm{~mm}$ ). C and D: Postoperative images showing that only one-quarter of the tumor has been removed through the right OTA with the transfalcine approach. The areas with color indicate neuronal fibers.

hemispheric side because the right occipital lobe leaned against the falx. The left transverse sinus was ligated and divided, the tentorium was incised up to the tentorial incisura, and the tumor in the infratentorial space was removed. Tumor devascularization and debulking were successfully achieved, but because there were several blind spots behind the falx and tentorium, tumor around the vein of Galen and the straight sinus was left (Fig. 3C and D). After the operation, the patient recovered well without sequelae. The histological diagnosis was a transitional meningioma (Ki-67 9.4\%, WHO grade I). The second operation was performed 2 months later via a right occipital bitranstentorial/falcine approach with the patient in the lateral semiprone position. The tumor was not very hemorrhagic due to devascularization performed at the first operation. The tumor was removed with ease because of the surgical spaces around the tumor, with the exception of the small residual tumor around the vein of Galen and straight sinus (Fig. 3E and F). Postoperative 3D CT venography showed patency of the deep venous system and disappearance of engorgement of the internal cerebral veins. The patient was symptom free (mRS score 0 ) without tumor recurrence at 1 year after the last operation.

\section{Triple-Staged Operation}

Case 4

A 53-year-old woman gradually developed cognitive dysfunction and memory disturbance and was referred to our institution. The patient exhibited right homonymous
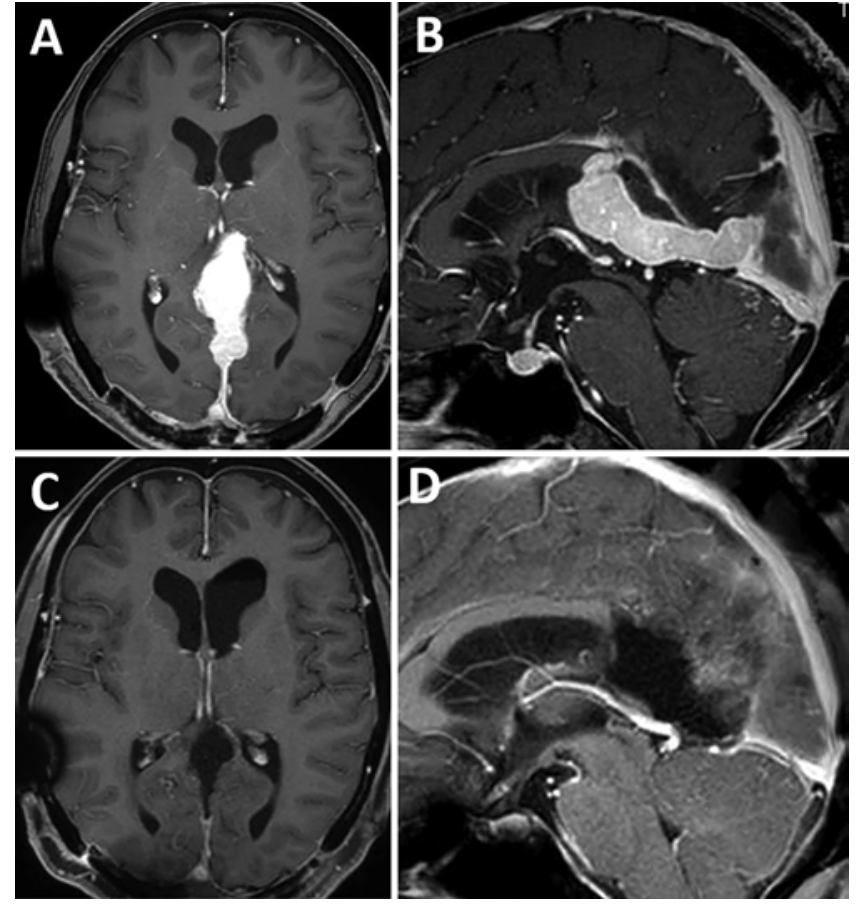

FIG. 5. Case 4. Axial ( $A$ and $C$ ) and sagittal (B and D) Gd-enhanced T1-weighted MR images. A and B: Postoperative images showing the tumor remnant in the left lateral ventricle and around the straight sinus after removal through the left OTA with the transfalcine approach. $\mathbf{C}$ and D: Postoperative images showing gross-total removal of the tumor through the right parietal interhemispheric transcallosal approach.

hemianopia. MRI showed a large pineal region tumor (57 $\mathrm{mm}$ in maximum diameter), which was compressing the bilateral thalamus and midbrain (Fig. 4A and B). Obstructive hydrocephalus was present. The bilateral internal cerebral veins and vein of Galen were compressed downward and only faintly visualized but still flowed into the thin straight sinus. The bilateral basal veins formed the collateral supply for the internal cerebral veins. The first operation was conducted using the right OTA with the transfalcine approach, with the patient in the lateral semiprone position. After incisions of the tentorium and falx were made, tumor debulking was performed, but massive bleeding persisted. Only one-quarter of the tumor was removed (Fig. 4C and D). After the operation, left lower quadrantanopia occurred, and the patient's hydrocephalus worsened. A ventriculoperitoneal shunt was placed, and the left lower quadrantanopia disappeared. The histological diagnosis was hemangiopericytoma (Ki-67 12\%, WHO grade II). Two months later, the second operation was performed via a left OTA with the transfalcine approach, with the patient in the lateral semiprone position. The tumor was subtotally removed, but postoperative MRI revealed a considerable tumor remnant around the vein of Galen to the straight sinus, extending into the left lateral ventricle (Fig. 5A and B). CyberKnife radiosurgery was performed, and the tumor size decreased slightly. Nine months later, the third operation was performed using the right parietal interhemispheric transcallosal approach with the patient in the lateral head-down position. The left lateral ventricle was opened through the partially resected splenium, and 
TABLE 1. Summary of multistaged, multidirectional approaches and outcomes of 4 cases of pineal region meningiomas

\begin{tabular}{|c|c|c|c|c|c|c|c|c|c|c|c|c|c|}
\hline $\begin{array}{l}\text { Case } \\
\text { No. }\end{array}$ & $\begin{array}{c}\text { Age } \\
\text { (yrs), } \\
\text { Sex }\end{array}$ & $\begin{array}{l}\text { Tumor } \\
\text { Size } \\
(\mathrm{mm})\end{array}$ & Approach & Side & $\begin{array}{c}\text { Op } \\
\text { Time } \\
\text { (mins) }\end{array}$ & Complications & $\begin{array}{c}\text { EOR } \\
\text { (final) }\end{array}$ & $\begin{array}{c}\text { MRI } \\
\text { Abnormality }\end{array}$ & $\begin{array}{l}\text { Radiation } \\
\text { Therapy }\end{array}$ & $\begin{array}{l}\text { Histological } \\
\text { Findings } \\
\text { (Ki-67\%) }\end{array}$ & $\begin{array}{l}\text { WHO } \\
\text { Grade }\end{array}$ & $\begin{array}{l}\mathrm{mRS} \\
\text { Score }\end{array}$ & $\begin{array}{c}\text { FU } \\
\text { (mos) }\end{array}$ \\
\hline 1 & $75, M$ & 31 & OTA & $\mathrm{Rt}$ & 401 & None & Gross total & None & None & Atypical & $\|$ & 0 & 13 \\
\hline \multirow[t]{2}{*}{2} & \multirow[t]{2}{*}{$47, \mathrm{~F}$} & \multirow[t]{2}{*}{41} & OBT/FA & $\mathrm{Rt}$ & 687 & Transient VFD & & & None & Fibrous & I & & 52 \\
\hline & & & OTA & $\mathrm{Lt}$ & 505 & None & Gross total & None & & & & 0 & \\
\hline \multirow[t]{2}{*}{3} & \multirow[t]{2}{*}{$65, \mathrm{M}$} & \multirow[t]{2}{*}{54} & CSITSA & $\mathrm{Lt}$ & 1152 & None & & & CyberKnife & Transitional (9.4) & I & & 15 \\
\hline & & & OBT/FA & $\mathrm{Rt}$ & 463 & None & Subtotal & None & & & & 0 & \\
\hline \multirow[t]{3}{*}{4} & \multirow[t]{3}{*}{$53, \mathrm{~F}$} & \multirow[t]{3}{*}{57} & OTA w/ TF & Rt & 708 & Transient VFD & & & CyberKnife & HPC (12) & II & & 38 \\
\hline & & & OTA w/ TF & $\mathrm{Lt}$ & 709 & None & & & & & & & \\
\hline & & & СРТCA & $\mathrm{Rt}$ & 438 & None & Gross total & None & & & & 2 & \\
\hline
\end{tabular}

CPTCA = contralateral parietal interhemispheric transcallosal approach; CSITSA = combined supra-linfratentorial transsinus approach; EOR = extent of resection; $\mathrm{FU}=$ follow-up; HPC = hemangiopericytoma; OBT/FA = occipital bitranstentorial/falcine approach; TF = transfalcine approach; VFD = visual field deficit (homonymous hemianopia).

the anterior end of the tumor was exposed in the left lateral ventricle. The tumor was totally removed (Fig. 5C and D). Postoperative 3D CT venography showed patency of the deep venous system. The patient's right homonymous hemianopia and cognitive dysfunction, which were present preoperatively, persisted, but without tumor recurrence at 1 year after the last operation (mRS score 2).

\section{Results}

Table 1 summarizes the clinical characteristics of the 4 patients with meningiomas in the pineal region. The mean operative time was 737 minutes (range 401-1152 minutes) at the first operation and 559 minutes (range 463-709 minutes) at the second operation. All tumors were grosstotally removed except for one (subtotal), and there was no permanent surgical morbidity. Transient homonymous quadrantanopia occurred after 2 of the 8 operations in the 4 patients (25\%). Postoperative MRI showed neither abnormal findings nor recurrence at 1-4 years after the operations. The patency of the deep venous system was preserved in all patients. Two patients (cases 3 and 4) underwent CyberKnife therapy after the operation. During the follow-up period (mean 29.5 months [range 13-52 months]), there were no recurrences after the final operation.

\section{Discussion}

We treated 4 consecutive cases of small to large meningiomas in the pineal region. The 3 large tumors (maximum diameter $>40 \mathrm{~mm}$ ) were gross-totally or subtotally removed without permanent operation-related morbidity using multistaged, multidirectional approaches with gravity retraction of the occipital lobe with the patients in the lateral semiprone position. An intentional initial large horseshoe occipital scalp incision was helpful for the next operation performed from the opposite side.

Surgical approaches to pineal region tumors are generally divided into 2 major approaches: the supracerebellar infratentorial approach and the OTA. The supracerebellar infratentorial approach might be chosen for pineal region meningiomas originating from underneath the tentorium near the junction of the vein of Galen with the straight sinus and displacing the galenic venous system anteriorly and upward .,11 However, most pineal region meningiomas grow downward from the origin and are located below the falcotentorial junction, and they displace the galenic venous system downward. ${ }^{10}$ Pineal region meningiomas, which originate near the falcotentorial junction, receive blood supply from the meningeal arteries along the tentorium and falx, and tend to grow in both infra- and supratentorial spaces with lateral extension.

For these reasons, the OTA is preferable in most cases, because of the access to the pineal region without sacrificing the bridging veins from the cerebellar tentorial surface, early devascularization of the feeding arteries along the tentorium and falx, and exposure of both the supra- and infratentorial spaces. ${ }^{2,9,10,15,16}$ However, pineal region meningiomas, especially large ones, tend to be separated into 4 anatomical compartments by the cerebral falx, bilateral tentorium, and cerebellar falx. Furthermore, the straight sinus is located in the junction between the falx and tentorium and receives venous blood from the bilateral internal cerebral veins, vein of Galen, bilateral basal veins, and internal occipital veins. Consequently, meningiomas in this area involve these vital deep venous systems and sometimes develop collateral venous channels. ${ }^{4,9}$ The difficulty with surgical removal of tumors in the pineal region depends mainly on the anatomical relationships between the tumor and the deep venous system and its collateral venous channels. ${ }^{4,17}$ Indeed, injury to the galenic venous system and/or its collateral venous channels during tumor removal may cause postoperative venous infarction with formidable morbidity. 4,9 These particular anatomical and venous conditions may prevent safe total removal of pineal region meningiomas via the unilateral OTA, especially in cases of large tumors greater than $4 \mathrm{~cm}$ in diameter. ${ }^{15}$

Visual field deficits are a major postoperative morbidity associated with pineal tumor removal via the occipital interhemispheric approach. Simultaneous combined supra-/ infratentorial approaches via the bilateral occipital lobes for falcotentorial meningioma removal in the prone position has resulted in postoperative transient visual field 

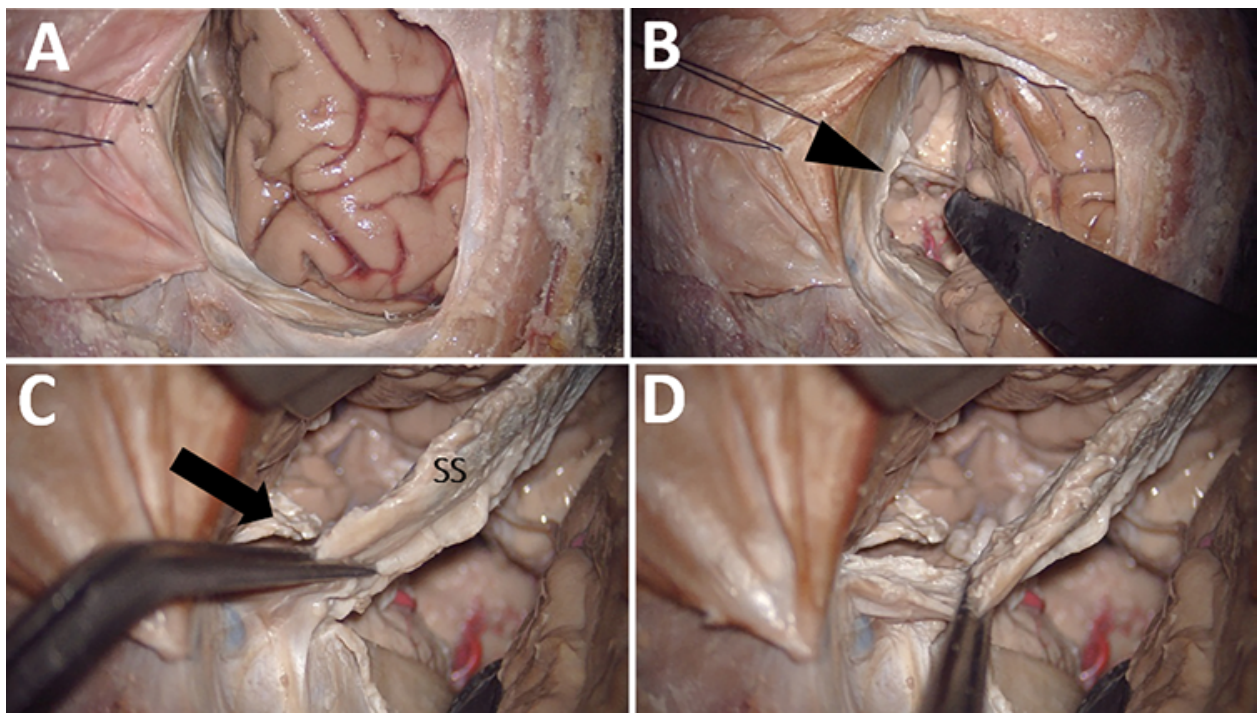

FIG. 6. Photographs of cadaveric study of the OTA and occipital bitranstentorial/falcine approach. A: The right occipital lobe is retracted by gravity in the right-side down, lateral semiprone position. B: OTA. The right tentorium is incised along the straight sinus (arrowhead). C and D: Occipital bitranstentorial/falcine approach. The arrow indicates the incised contralateral tentorium through the incised falx. A blind spot remains due to incomplete mobilization of the straight sinus (SS).

deficits in $100 \%$ of patients, including cortical blindness in $83 \% .{ }^{16}$ Although most of these sequelae resolve quickly, unilateral OTA in the semisitting position causes complete or partial hemianopia in $30 \%-100 \%$ of patients immediately after the operation. ${ }^{9,12}$ The incidence of a postoperative visual field deficit (13\%) was reduced by introducing the three-quarter prone position (lateral semiprone position) for gravity retraction of the occipital lobe..$^{15} \mathrm{To}$ minimize occipital lobe retraction, we also used the gravity retraction technique in the lateral semiprone position in all cases. ${ }^{1}$ However, postoperative transient hemianopia occurred after 2 of the 8 operations performed in our 4 patients. Therefore, the simultaneous bilateral occipital approach in the prone position cannot avoid cortical blindness. The occipital bitranstentorial/falcine approach via the unilateral occipital interhemispheric space has several advantages for access to the tumor in the contralateral infratentorial space and allows for easier observation of the contralateral basal vein. ${ }^{5,8}$ Although this approach has the advantages of both the unilateral OTA and bilateral combined supra- and infratentorial approaches, blind spots remain due to incomplete skeletonization and incomplete mobilization of the straight sinus, and management of the contralateral basal vein after the final stage of the tumor removal carries some risks (Fig. 6). Meningiomas in the pineal region tend to be larger than other malignant pineal tumors at the time of discovery, which suggests that observation and safe total removal are likely to be difficult in a single operation. ${ }^{15}$

Given that meningiomas have a biologically benign nature, our basic policy for safe removal of these tumors in the pineal region does not emphasize total removal in a single operation but rather planning of multistaged, multidirectional surgery. The intentional initial large occipital scalp incision extending to both sides helps when the next operation is performed from the contralateral side. There was less tumor bleeding during the second operation than during the first operation due to previous devascularization and easier dissection of the reduced tumor from the surrounding vital structures without causing deep vein injury. The staged bilateral approaches to the galenic venous complex enabled us to dissect veins from the tumor safely. If postoperative hemianopia occurs during the first operation, the next operation should be delayed until it resolves to avoid cortical blindness. Our multistaged, multidirectional strategy can help achieve safe tumor removal. Therefore, although the multiple operations are a burden to the patient, we think that a single-stage operation should not be used to treat challenging benign meningiomas of the pineal region.

\section{Conclusions}

A multistaged, multidirectional strategy with an intentionally large occipital scalp incision and gravity retraction of the occipital lobe is a good choice for the safe removal of large meningiomas in the pineal region.

\section{References}

1. Ausman JI, Malik GM, Dujovny M, Mann R: Three-quarter prone approach to the pineal-tentorial region. Surg Neurol 29:298-306, 1988

2. Bassiouni H, Asgari S, König HJ, Stolke D: Meningiomas of the falcotentorial junction: selection of the surgical approach according to the tumor type. Surg Neurol 69:339-349, 2008

3. Davies J, Tawk RG, Lawton MT: The contralateral transcingulate approach: operative technique and results with vascular lesions. Neurosurgery 71 (1 Suppl Operative):4-14, 2012

4. Goto T, Ohata K, Morino M, Takami T, Tsuyuguchi N, Nishio A, et al: Falcotentorial meningioma: surgical outcome in 14 patients. J Neurosurg 104:47-53, 2006

5. Gusmão S, Oliveira MM, Arantes A, Ulhoa TH, Morato EG: Occipital bi-transtentorial/falcine approach for falcotentorial meningioma: case report. Arq Neuropsiquiatr 64:136-138, 2006 
6. Inoue A, Ohnishi T, Kohno S, Ohtsuka Y, Nakamura Y, Mizuno Y, et al: Two cases of pineal-region meningiomas derived from arachnoid membrane over the vein of Galen without dural attachment. World J Surg Oncol 13:226, 2015

7. Jamieson KG: Excision of pineal tumors. J Neurosurg 35:550-553, 1971

8. Kawashima M, Rhoton AL Jr, Matsushima T: Comparison of posterior approaches to the posterior incisural space: microsurgical anatomy and proposal of a new method, the occipital bi-transtentorial/falcine approach. Neurosurgery 51:1208-1221, 2002

9. Konovalov AN, Spallone A, Pitzkhelauri DI: Meningioma of the pineal region: a surgical series of 10 cases. J Neurosurg 85:586-590, 1996

10. Li Y, Zhao G, Wang H, Zhu W, Qu L, Li Y, et al: Use of 3Dcomputed tomography angiography for planning the surgical removal of pineal region meningiomas using Poppen's approach: a report of ten cases and a literature review. World J Surg Oncol 9:64, 2011

11. Lozier AP, Bruce JN: Meningiomas of the velum interpositum: surgical considerations. Neurosurg Focus 15(1):E11, 2003

12. Nazzaro JM, Shults WT, Neuwelt EA: Neuro-ophthalmological function of patients with pineal region tumors approached transtentorially in the semisitting position. J Neurosurg 76:746-751, 1992

13. Obrador S, Soto M, Gutierrez-Diaz JA: Surgical management of tumours of the pineal region. Acta Neurochir (Wien) 34:159-171, 1976

14. Poppen JL: The right occipital approach to a pinealoma. J Neurosurg 25:706-710, 1966

15. Qiu B, Wang Y, Ou S, Guo Z, Wang Y: The unilateral occipital transtentorial approach for pineal region meningiomas: a report of 15 cases. Int J Neurosci 124:741-747, 2014

16. Quinones-Hinojosa A, Chang EF, McDermott MW: Falco- tentorial meningiomas: clinical, neuroimaging, and surgical features in six patients. Neurosurg Focus 14(6): e11, 2003

17. Samii M, Carvalho GA, Tatagiba M, Matthies C, Vorkapic $\mathrm{P}$ : Meningiomas of the tentorial notch: surgical anatomy and management. J Neurosurg 84:375-381, 1996

18. Sekhar LN, Goel A: Combined supratentorial and infratentorial approach to large pineal-region meningioma. Surg Neurol 37:197-201, 1992

19. Stein BM: Surgical treatment of pineal tumors. Clin Neurosurg 26:490-510, 1979

20. Zaidi HA, Chowdhry SA, Nakaji P, Abla AA, Spetzler RF: Contralateral interhemispheric approach to deep-seated cavernous malformations: surgical considerations and clinical outcomes in 31 consecutive cases. Neurosurgery 75:80-86, 2014

21. Ziyal IM, Sekhar LN, Salas E, Olan WJ: Combined supra/ infratentorial-transsinus approach to large pineal region tumors. J Neurosurg 88:1050-1057, 1998

\section{Disclosures}

The authors report no conflict of interest concerning the materials or methods used in this study or the findings specified in this paper.

\section{Author Contributions}

Conception and design: Otani. Acquisition of data: Wada, Takeuchi. Analysis and interpretation of data: Tomiyama. Statistical analysis: Toyooka. Study supervision: Mori.

\section{Correspondence}

Kentaro Mori: National Defense Medical College, Saitama, Japan. kmori@ndmc.ac.jp. 